OPEN ACCESS

Edited by:

Maoli Duan,

Karolinska Institutet, Sweden

Reviewed by:

Yong Feng,

University of South China Affiliated

Changsha Central Hospital, China

Hongyang Wang,

People's Liberation Army General

Hospital, China

"Correspondence:

Yanmei Feng

ymfeng@sjtu.edu.cn

Zhen Zhang

zhangzhen1994s@outlook.com

Di Qian

skeayqd@sina.com

${ }^{\dagger}$ These authors have contributed equally to this work and share first authorship

Specialty section:

This article was submitted to

Clinical Diabetes,

a section of the journal

Frontiers in Endocrinology

Received: 23 August 2021

Accepted: 27 October 2021

Published: 18 November 2021

Citation:

Shen Y, Zheng Z, Xiao L, Liu C,

Guo J, Chen Z, Wu Y, Shi H, Zhang $Z$, Qian $D$, Feng $Y$ and Yin $S$ (2021) Association of Glycosylated

Hemoglobin A1c Level With

Sudden Sensorineural Hearing

Loss: A Prospective Study.

Front. Endocrinol. 12:763021. doi: 10.3389/fendo.2021.763021

\section{Association of Glycosylated Hemoglobin A1c Level With Sudden Sensorineural Hearing Loss: A Prospective Study}

\author{
Ying Shen ${ }^{1,2 \dagger}$, Zhong Zheng ${ }^{1,2+}$, Lili Xiao ${ }^{1,2}$, Chengqi Liu ${ }^{1,2}$, Jingyi Guo ${ }^{3}$, \\ Zhengnong Chen ${ }^{1,2}$, Yaqin Wu ${ }^{1,2}$, Haibo Shi ${ }^{1,2}$, Zhen Zhang ${ }^{1,2 *}$, Di Qian ${ }^{4 *}$, \\ Yanmei Feng ${ }^{1,2 *}$ and Shankai Yin ${ }^{1,2}$ \\ 1 Department of Otolaryngology Head and Neck Surgery, Shanghai Jiao Tong University Affiliated Sixth People's Hospital, \\ Shanghai, China, 2 Shanghai Key Laboratory of Sleep Disordered Breathing, Shanghai, China, ${ }^{3}$ Clinical Research Center, \\ Shanghai Jiao Tong University Affiliated Sixth People's Hospital, Shanghai, China, ${ }^{4}$ Department of Otolaryngology, People's \\ Hospital of Longhua, Shenzhen, China
}

Glycosylated hemoglobin A1c (HbA1c) level has strong relevance to microvascular disorders, which are also thought to be the current main aspect of sudden sensorineural hearing loss (SSNHL), so we aim to elucidate the association of the HbA1c level with the severity, types, and prognosis of SSNHL. In this study, comparative analyses based on propensity score matching of the severity, types, and prognosis of SSNHL with the HbA1c level in 116 patients diagnosed as SSNHL were conducted, where they were divided into diabetes mellitus (DM) group and non-DM group. We finally found that, among patients with SSNHL, diabetic patients had a higher HbA1c level, more severe hearing loss, and poorer prognosis than non-diabetic patients. The HbA1c level was found to be significantly correlated with the severity and types of SSNHL, while no strong relevance was found between the higher $\mathrm{HbA1c}$ level and the poorer prognosis of SSNHL.

Keywords: sudden sensorineural hearing loss, diabetes mellitus, glycosylated hemoglobin A1c, microvascular disorders, propensity score matching

\section{INTRODUCTION}

Sudden sensorineural hearing loss (SSNHL) is generally defined as a hearing loss that is sensorineural in nature. It occurs within a 72 -h window and consists of a decrease in hearing of greater than $30 \mathrm{~dB}$, affecting at least three consecutive frequencies (1). It has a prevalence of five to 27 individuals per 100,000 people in the United States (2). The main pathogenic causes of SSNHL are still unidentified, yet several pathophysiological mechanisms have been proposed, including viral infection, vascular disorders, autoimmune disease, and other causes, such as cochlear membrane rupture (3). 
Among the various possible etiologies and pathogenesis, mainstream pathophysiological mechanisms for SSNHL attribute its occurrence to microvascular disorders. Microvascular damage as well as other microcirculatory disturbances involving sudden increases in blood viscosity, along with embolic and thrombotic episodes (4) such as within the labyrinthine artery, can interrupt the vascular supply to the cochlea, eventually causing cochlea dysfunction and resulting in SSNHL.

Diabetes mellitus (DM) contributes the greatest risks to microvascular disorders. Diabetic kidney diseases, diabetic retinopathy, and diabetic foot are a few typical long-term microvascular complications of DM patients resulting from extended poorly controlled high blood sugar levels. Accounting for $90-95 \%$ of all DM, type 2 diabetes mellitus (T2DM) frequently goes undiagnosed for many years as hyperglycemia develops gradually. The American Diabetes Association states that even undiagnosed T2DM patients are at an increased risk of developing both macrovascular and microvascular complications (5).

The glycosylated hemoglobin A1c (HbA1c) level has been widely recognized as a reliable diagnostic biomarker for DM, especially for T2DM. About $6 \%$ of hemoglobin A can be categorized as glycated hemoglobin (6) and, among those, HbA1c is composed of approximately $5 \%$ glycated hemoglobin in a healthy individual (7). It forms when glucose attaches specifically to the $\mathrm{N}$-terminal valine of the $\beta$ subunit of hemoglobin and reflects the average serum glucose concentration over the lifespan of an erythrocyte, which is on average 120 days (8). Thus, HbAlc testing is helpful for clinicians to assess the long-term glucose control status of the patient. A previous study has shown that, for every $1 \%$ rise in $\mathrm{HbA} 1 \mathrm{c}$ level, there is a $37 \%$ increase in the risk of a microvascular disease (9). Although many studies have highlighted the negative role of DM in the pathogenesis and prognosis of SSNHL, few studies have explored the relationship between HbA1c and SSNHL. The present study is designed to elucidate the association of the HbA1c level with the severity, types, and prognosis of SSNHL in a group of well-defined patients.

\section{MATERIALS AND METHODS}

\section{Study Population}

We performed a prospective study of patients admitted to the Department of Otolaryngology Head and Neck Surgery at Shanghai Jiao Tong University Affiliated Sixth People's Hospital, Shanghai, China, with a diagnosis of unilateral SSNHL from August 1, 2018 through December 31, 2020. All patients met the criteria: sensorineural in nature, occurred within a $72-\mathrm{h}$ window, and decrease in hearing of greater than $30 \mathrm{~dB}$ affecting at least three consecutive frequencies; unilateral onset; age of 18 years or older; examined less than 3 weeks after the onset of disease; and not receiving a previous therapy. Patients with any accompanying disease or trauma that contributes to hearing loss, such as Meniere's disease, vestibular schwannoma, or other central nervous system disease, head injury, or use of ototoxic medicine were excluded. Eventually, a total of 116 patients were enrolled in our research. The diagnostic criteria for DM were based on the report of the expert committee on the diagnosis and classification of diabetes mellitus (10), and the participants were divided into two groups with SSNHL: the DM group and the non-DM group according to their previous medical history and blood tests for DM.

\section{Measurements and Treatment Process}

All patients underwent general clinical interviews, laboratory testing, otoscopic examinations, complete audiologic tests, including pure-tone audiometry, tympanogram tests, and otoacoustic emission measures. Internal auditory canal magnetic resonance imaging was also conducted to exclude retrocochlear pathology, and each patient was provided with personal protection devices such as ear cover and earplug during the inspection.

Auditory evaluations were mainly determined by puretone average (PTA) from $125 \mathrm{~Hz}$ to $8 \mathrm{kHz}$ through measuring air and bone conduction, which were all performed by certified and experienced audiologists. Puretone audiometry was conducted in sound-proofed rooms with noise less than $30 \mathrm{~dB}(\mathrm{~A})$ using a calibrated GSI-61TM audiometer (United States) coupled with TDH 39 headphones. The hearing thresholds of all SSNHL patients were expressed as the mean hearing level of impaired frequencies. According to the report of the First Informal Consultation on Future Programme Developments for WHO$\mathrm{PDH}, 1997$ (11), hearing was defined as normal if PTA was less than $25 \mathrm{~dB} \mathrm{HL}$, and the severity of hearing loss was defined as "mild" if PTA was $26 \mathrm{~dB}$ HL but less than $40 \mathrm{~dB} \mathrm{HL}$, "moderate" if it was greater than $41 \mathrm{~dB}$ HL but less than $60 \mathrm{~dB}$ $\mathrm{HL}$, "severe" if it was greater than $61 \mathrm{~dB}$ HL but less than 80 $\mathrm{dB}$ HL, and "profound" if it was greater than $81 \mathrm{~dB}$ HL. Furthermore, we determined the different types of SSNHL according to the Guidelines for Diagnosis and Treatment of Sudden Deafness, 2015, China (12), which are judged by the audiometric configurations of patients, including their impaired frequencies and degree of hearing loss. It was classified as "low-frequency descending type of SSNHL (LFSSNHL)" if hearing loss happened at frequencies below and including $1 \mathrm{kHz}$, "high-frequency descending type of SSNHL (HF-SSNHL)" if hearing loss happened at frequencies above and including $2 \mathrm{kHz}$, "flat type of SSNHL (FT-SSNHL)" if hearing loss happened at all frequencies and the mean hearing level was less than or equal to $80 \mathrm{~dB} \mathrm{HL}$, and "total deafness of SSNHL (TD-SSNHL)" if the mean hearing level was greater than or equal to $81 \mathrm{~dB} \mathrm{HL}$.

For laboratory testing, all patients had fast blood samples taken in the morning after $8 \mathrm{~h}$ of fasting, which included fasting plasma glucose $(\mathrm{mmol} / \mathrm{L})$, glycosylated hemoglobin A1c $(\mathrm{HbA} 1 \mathrm{c}, \%)$, serum creatinine $(\mu \mathrm{mol} / \mathrm{L}), \mathrm{D}-\mathrm{D}$ dimer $(\mathrm{mg} / \mathrm{L}$ fibrinogen equivalent unit), total cholesterol $(\mathrm{mmol} / \mathrm{L})$, triglycerides $(\mathrm{mmol} / \mathrm{L})$, high-density lipoprotein $(\mathrm{mmol} / \mathrm{L})$, and low-density lipoprotein $(\mathrm{mmol} / \mathrm{L})$. Thereafter, the HbAlc test was performed using a certified method by the NGSP (www. 
ngsp.org), reported in \%HbAlc. The analyses of all blood samples were performed by routine laboratory screening in our hospital central laboratory.

After admission, all patients were treated with comprehensive treatments, including the use of systemic steroids with initial doses of methylprednisolone at $80 \mathrm{mg}$ intravenously, oral antioxidants, and neurotrophic drugs. Patients with "FTSSNHL" and "TD-SSNHL" took an additional drug of batroxobin which lowers fibrinogen, with an initial dose of 10 $\mathrm{BU}$ and then $5 \mathrm{BU}$ for subsequent administrations according to the Guidelines for Diagnosis and Treatment of Sudden Deafness, 2015, China (12). The serum fibrinogen level was monitored before each use to confirm it is higher than $1 \mathrm{~g} / \mathrm{L}$ to avoid bleeding and other related side effects. During the treatments, blood glucose was monitored regularly through fixed-point monitoring of blood glucose from fingerstick testing for five times a day that covered timepoints of after fasting, $2 \mathrm{~h}$ after three meals, and at $21 \mathrm{PM}$. When the blood glucose of a patient fluctuated, an endocrinologist would be consulted in time and modify his hypoglycemic treatments. Furthermore, patients whose blood glucose fluctuates greatly or was difficult to control had their systemic steroids therapy dosage modified or even stopped immediately. It should be stressed that we defined treatment course here as the duration of systemic steroids. All SSNHL patients would receive treatments of antioxidants and neurotrophic agents for 2 weeks.

We were able to obtain follow-up auditory evaluations for each patient on day 14 after treatments. The prognosis of SSNHL was judged by the post-treatment hearing thresholds of PTA in impaired frequencies. Treatment was deemed effective if the PTA of the impaired frequencies improved by at least $15 \mathrm{~dB}$ or when the damaged ear achieved the same level as the normal or unaffected ear of the patient. It was deemed ineffective in the treatment of SSNHL if the PTA of the impaired frequencies improved by less than $15 \mathrm{~dB}$ (12).

\section{Statistical Analysis}

Categorical variables were expressed as number and percentage. Data were investigated using Kolmogorov-Smirnov test to determine the distribution pattern. The data of clinical character did not have a normal distribution $(P<0.05)$; thus, quantitative variables were presented as median (inter-quartile range, IQR). We adopted a non-parametric test (Mann-Whitney $U$-test) for quantitative variables and $\chi^{2}$ test or Fisher's exact test for categorical variables. Correlations between HbA1c level and variables were sought using the Spearman rank correlation test. Univariate analysis was carried out to assess differences in demographic, clinical, and metabolic variables, accompanying symptoms, severity of hearing loss, and types of SSNHL between diabetic and non-diabetic groups. To overcome selection biases in this research, we performed a 1:1 match for each patient by using propensity score matching (PSM). A two-sided $p$-value $<0.05$ was taken to indicate statistical significance. All statistical analyses were performed with the aid of SPSS version 26 (SPSS, USA).

\section{RESULTS}

\section{Clinical Characteristics of Patients Before PSM}

Of the 116 SSNHL patients enrolled (58 men [50.0\%]; 58 women [50.0\%]; median (IQR) age, 54.00 [25.25] years), 31 patients had DM (DM group) and 85 were non-diabetic (non-DM group). The course of DM in these 31 diabetic patients was 10.89 [6.89] years on average. The clinical characteristics of the study populations are presented in Table 1. There were no statistically significant differences in gender, time to treatment initiation, the side of the affected ear, tinnitus and vertigo associated incidence, admission systolic blood pressure, admission diastolic blood pressure, serum creatinine, D-D dimer, total cholesterol, triglycerides, high-density lipoprotein and low density lipoprotein between the two groups (all P > $0.05)$. We noted that diabetic patients were much older than non-diabetic ones (61.00 [15.00] vs 49.00 [26.00] years, $\mathrm{P}<0.05)$. Meanwhile, the FBG (10.85 [6.89] vs 6.19 [1.91] mmol/L) and HbA1c level (7.30 [1.50] vs 5.60 [0.40] \%) (both $\mathrm{P}<0.05)$ were higher in the diabetic patients. However, it was worth mentioning that steroid medications had glycemic effects and thus FBG might been greatly affected by using steroid medications in a short time. We did not included FBG in the subsequent analysis.

Besides, diabetic patients' pre-treatment PTA of the impaired frequencies was higher than non-diabetic patients (91.67 [19.17] vs 66.67 [37.38] dBHL, $\mathrm{P}<0.05)$ and among all types of SSNHL, "total deafness type of SSNHL" dominated. We further compared the degree of the impaired frequencies' hearing loss between the two groups and found that the differences in the proportion of "mild to moderate" and "severe to profound" hearing loss were statistically significant $(\mathrm{P}<0.05)$ (as shown in Table 1). Meanwhile, the treatment course for patients with DM was also shorter than those without DM $(\mathrm{P}<0.05)$, mainly due to worse blood glucose control in diabetic SSNHL patients.

While focusing on the prognosis of SSNHL, we found that post-treatment PTA of the impaired frequencies was much higher in DM group (80.83 [35.00] vs 42.86 [43.72] $\mathrm{dBHL}, \mathrm{P}<0.05)$. The number of effective cases of non$\mathrm{DM}$ group was also significantly higher than that of DM group $(\mathrm{P}<0.05)$. Only $32.3 \%$ diabetic SSNHL patients in our research performed effectively in their treatment (as shown in Table 1).

\section{Pathogenic Features in the Matched Data Set After PSM Using "Age" as the Predictor}

Considering the significant difference in age, we performed PSM and used "age" as the predictor here to correct for potential bias and further explore the pathogenic features between the two groups, mainly focusing on their level of $\mathrm{HbAlc}$, the severity of hearing loss, and the characteristics of their audiometric configuration. After PSM, 26 pairs of patients were successfully matched (Table 2). It was worth noting that the HbA1c level in the DM group was still significantly higher than that in the non-DM group after PSM [7.40 (2.13) vs. $5.70(0.60) \%, P<0.05)$. The diabetic SSNHL patients 
TABLE 1 | Clinical characteristics of study participants before propensity score matching.

\begin{tabular}{|c|c|c|c|}
\hline Indicators & DM group $(n=31)$ & Non-DM group $(n=85)$ & $P$-value \\
\hline \multicolumn{4}{|l|}{ Demographics } \\
\hline $\begin{array}{l}\text { Gender } \\
\text { (male/female) }\end{array}$ & $16: 15$ & $42: 43$ & 0.834 \\
\hline $\begin{array}{l}\text { Age } \\
\text { (years) }\end{array}$ & $61.00(15.00)$ & $49.00(26.00)$ & $<0.001^{*}$ \\
\hline $\begin{array}{l}\text { Time to treatment initiation } \\
\text { (days) }\end{array}$ & $5.00(5.00)$ & $4.00(5.00)$ & 0.616 \\
\hline $\begin{array}{l}\text { Side } \\
\text { (left/right) }\end{array}$ & $15: 16$ & $43: 42$ & 0.834 \\
\hline \multicolumn{4}{|l|}{ Clinical and metabolic variables } \\
\hline $\begin{array}{l}\mathrm{SBP} \\
(\mathrm{mmHg})\end{array}$ & $129.00(22.00)$ & $122.00(15.00)$ & 0.067 \\
\hline $\begin{array}{l}\mathrm{DBP} \\
(\mathrm{mmHg})\end{array}$ & $76.00(15.00)$ & $78.00(14.00)$ & 0.726 \\
\hline $\begin{array}{l}\mathrm{BMI} \\
\left(\mathrm{kg} / \mathrm{m}^{2}\right)\end{array}$ & $22.72(5.62)$ & $23.42(4.00)$ & 0.925 \\
\hline $\begin{array}{l}\text { FBG } \\
(\mathrm{mmol} / \mathrm{L})\end{array}$ & $10.85(6.89)$ & $6.19(1.91)$ & $<0.001^{*}$ \\
\hline $\begin{array}{l}\mathrm{HbA1c} \\
(\%)\end{array}$ & $7.30(1.50)$ & $5.60(0.40)$ & $<0.001^{*}$ \\
\hline $\begin{array}{l}\text { Scr } \\
\text { (umol/L) }\end{array}$ & $59.00(21.50)$ & $59.00(18.00)$ & 0.849 \\
\hline $\begin{array}{l}\text { DD } \\
(\mathrm{mg} / \mathrm{L} \text { FEU) }\end{array}$ & $0.16(0.15)$ & $0.17(0.13)$ & 0.886 \\
\hline $\begin{array}{l}\text { TC } \\
(\mathrm{mmol} / \mathrm{L})\end{array}$ & $5.20(0.82)$ & $5.11(1.31)$ & 0.757 \\
\hline $\begin{array}{l}\text { TG } \\
(\mathrm{mmol} / \mathrm{L})\end{array}$ & $0.99(0.54)$ & $0.90(0.94)$ & 0.107 \\
\hline $\begin{array}{l}\mathrm{HDL} \\
(\mathrm{mmol} / \mathrm{L})\end{array}$ & $1.36(0.37)$ & $1.38(0.53)$ & 0.135 \\
\hline $\begin{array}{l}\mathrm{LDL} \\
(\mathrm{mmol} / \mathrm{L})\end{array}$ & $3.05(1.05)$ & $3.01(1.19)$ & 0.898 \\
\hline $\begin{array}{l}\text { Pre-treatment PTA of the impaired frequencies } \\
(\mathrm{dBHL})\end{array}$ & 91.67 (19.17) & 66.67 (37.38) & $<0.001^{*}$ \\
\hline $\begin{array}{l}\text { Treatment course } \\
\text { (days) }\end{array}$ & $5.00(2.50)$ & $5.00(0)$ & $0.011^{*}$ \\
\hline $\begin{array}{l}\text { Post-treatment PTA of the impaired frequencies } \\
(\mathrm{dBHL})\end{array}$ & $80.83(35.00)$ & $42.86(43.72)$ & $<0.001^{*}$ \\
\hline Effective case $(n, \%)$ & $10(32.3 \%)$ & $50(58.8 \%)$ & $0.011^{*}$ \\
\hline \multicolumn{4}{|l|}{ Accompanying symptoms $(n, \%)$} \\
\hline Vertigo & $17(56.7 \%)$ & $36(42.4 \%)$ & 0.232 \\
\hline Tinnitus & $26(86.7 \%)$ & 72 (84.7\%) & 0.912 \\
\hline \multicolumn{4}{|l|}{ Severity of hearing loss ( $n, \%)$} \\
\hline Mild to moderate & $3(9.7 \%)$ & 37 (43.5\%) & $0.001^{*}$ \\
\hline Severe to profound & $28(90.3 \%)$ & $48(56.5 \%)$ & \\
\hline \multicolumn{4}{|l|}{ Audiometric configurations ( $n, \%)$} \\
\hline LF-SSNHL & $1(3.2 \%)$ & $10(11.8 \%)$ & $<0.001^{*}$ \\
\hline HF-SSNHL & $2(6.5 \%)$ & $4(4.7 \%)$ & \\
\hline FT-SSNHL & 4 (12.9\%) & 44 (51.8\%) & \\
\hline TD-SSNHL & $24(77.4 \%)$ & 27 (31.8\%) & \\
\hline
\end{tabular}

DM, diabetes mellitus; SBP, admission systolic blood pressure; DBP, admission diastolic blood pressure; BMI, body mass index; FBG, fasting plasma glucose; HbA1C, glycosylated hemoglobin A1C; Scr, serum creatinine; DD, D-D dimer; TC, total cholesterol; TG, triglycerides; HDL, high-density lipoprotein; LDL, low-density lipoprotein; PTA, pure-tone average;

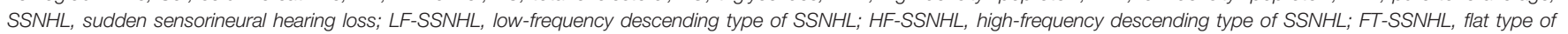
SSNHL; TD-SSNHL, total deafness of SSNHL.

${ }^{*} p<0.05$ (statistically significant).

The bold values in "Effective cases ( $n$,\%)" were the number or percentage of SSNHL patients who were classified as effective according to the outcome evaluation criteria.

The bold values in "P values" mean the statistical indicators that were taken as significant when smaller than 0.05.

performed much more poorly in pre-treatment PTA of the impaired frequencies than non-diabetic SSNHL patients [93.08 (19.17) vs. 76.43 (35.37) dBHL, $P<0.05$ ]. The degree of hearing loss of the impaired frequencies and the audiometric configurations of SSNHL between the two groups also show significant differences $(P<0.05)$. Specifically, "FT-SSNHL" dominated in SSNHL patients without DM, while "TD-SSNHL" was the most common type in diabetic SSNHL patients (as shown in Table 2). 
TABLE 2 | Pathogenic features in the matched data set after propensity score matching using "age" as the predictor.

\begin{tabular}{|c|c|c|c|}
\hline Indicators & DM group $(n=26)$ & Non-DM group $(n=26)$ & $P$-value \\
\hline \multicolumn{4}{|l|}{ Demographics } \\
\hline $\begin{array}{l}\text { Gender } \\
\text { (male/female) }\end{array}$ & $16: 10$ & $15: 11$ & 0.777 \\
\hline $\begin{array}{l}\text { Age } \\
\text { (years) }\end{array}$ & $59.50(11.50)$ & $59.00(11.50)$ & 0.934 \\
\hline $\begin{array}{l}\text { Time to treatment initiation } \\
\text { (days) }\end{array}$ & $5.00(5.00)$ & $3.00(3.75)$ & 0.293 \\
\hline $\begin{array}{l}\text { Side } \\
\text { (left/right) }\end{array}$ & $13: 13$ & $9: 17$ & 0.262 \\
\hline \multicolumn{4}{|l|}{ Clinical and metabolic variables } \\
\hline $\begin{array}{l}\text { SBP } \\
(\mathrm{mmHg})\end{array}$ & $125.00(26.75)$ & $124.00(20.25)$ & 0.674 \\
\hline $\begin{array}{l}\mathrm{DBP} \\
(\mathrm{mmHg})\end{array}$ & $75.50(15.00)$ & $77.00(13.00)$ & 0.510 \\
\hline $\begin{array}{l}\mathrm{BMl} \\
\left(\mathrm{kg} / \mathrm{m}^{2}\right)\end{array}$ & $22.79(5.25)$ & $24.49(3.47)$ & 0.337 \\
\hline $\begin{array}{l}\mathrm{HbA1c} \\
(\%)\end{array}$ & $7.40(2.13)$ & $5.70(0.60)$ & $<0.001^{*}$ \\
\hline $\begin{array}{l}\text { Scr } \\
\text { (umol/L) }\end{array}$ & $58.50(21.25)$ & $62.50(15.00)$ & 0.252 \\
\hline $\begin{array}{l}\text { DD } \\
(\mathrm{mg} / \mathrm{L} \mathrm{FEU})\end{array}$ & $0.16(0.13)$ & $0.19(0.12)$ & 0.576 \\
\hline $\begin{array}{l}\text { TC } \\
(\mathrm{mmol} / \mathrm{L})\end{array}$ & $5.18(0.84)$ & $4.94(1.48)$ & 0.516 \\
\hline $\begin{array}{l}\text { TG } \\
(\mathrm{mmol} / \mathrm{L})\end{array}$ & $1.04(0.48)$ & $1.14(0.82)$ & 0.534 \\
\hline $\begin{array}{l}\mathrm{HDL} \\
(\mathrm{mmol} / \mathrm{L})\end{array}$ & $1.34(0.29)$ & $1.34(0.37)$ & 0.756 \\
\hline $\begin{array}{l}\mathrm{LDL} \\
(\mathrm{mmol} / \mathrm{L})\end{array}$ & $3.03(1.14)$ & $2.78(1.09)$ & 0.728 \\
\hline $\begin{array}{l}\text { Pre-treatment PTA of the impaired frequencies } \\
(\mathrm{dBHL})\end{array}$ & $93.08(19.17)$ & $76.43(35.37)$ & $0.011^{*}$ \\
\hline \multicolumn{4}{|l|}{ Accompanying symptoms ( $n, \%)$} \\
\hline Vertigo & $14(53.8 \%)$ & 12 (46.2\%) & 0.579 \\
\hline Tinnitus & $23(88.5 \%)$ & $20(76.9 \%)$ & 0.463 \\
\hline \multicolumn{4}{|l|}{ Severity of hearing loss $(n, \%)$} \\
\hline Mild to moderate & $2(7.7 \%)$ & $8(30.8 \%)$ & $0.035^{\star}$ \\
\hline Severe to profound & $24(92.3 \%)$ & $18(69.2 \%)$ & \\
\hline \multicolumn{4}{|l|}{ Audiometric configurations ( $n, \%)$} \\
\hline LF-SSNHL & 0 & $3(11.5 \%)$ & $0.042^{*}$ \\
\hline HF-SSNHL & $2(7.7 \%)$ & $2(7.7 \%)$ & \\
\hline FT-SSNHL & $4(15.4 \%)$ & $10(38.5 \%)$ & \\
\hline TD-SSNHL & $20(76.9 \%)$ & $11(42.3 \%)$ & \\
\hline
\end{tabular}

${ }^{*} p<0.05$ (statistically significant).

The bold values in "Effective cases ( $n, \%)$ " were the number or percentage of SSNHL patients who were classified as effective according to the outcome evaluation criteria.

The bold values in "P values" mean the statistical indicators that were taken as significant when smaller than 0.05.

DM, diabetes mellitus; SBP, admission systolic blood pressure; DBP, admission diastolic blood pressure; BMl, body mass index; HbA1C, glycosylated hemoglobin A1C; Scr, serum creatinine; DD, D-D dimer; TC, total cholesterol; TG, triglycerides; HDL, high-density lipoprotein; LDL, low-density lipoprotein; PTA, pure-tone average; SSNHL, sudden sensorineural hearing loss; LFSSNHL, low-frequency descending type of SSNHL; HF-SSNHL, high-frequency descending type of SSNHL; FT-SSNHL, flat type of SSNHL; TD-SSNHL, total deafness of SSNHL.

\section{Prognosis Conditions of SSNHL in the Matched Data Set After PSM Using Prognosis-Related Factors of SSNHL as the Predictors}

The prognosis for SSNHL recovery had been reported to be dependent on a number of factors, including the age of patients, presence of vertigo at onset, degree of hearing loss, audiometric configurations, treatment course, and time between onset of hearing loss and treatment (13-15). To remove biases, we performed another 1:1 match for each patient by using PSM where we used prognosis-related factors, including "age", "treatment course", "severity of hearing loss", and "audiometric configurations" as the predictors. Here 19 pairs of patients were eventually matched (as shown in Table 3). There were no statistically significant differences in age, course of sudden deafness, vertigo-associated incidence, pre-treatment PTA of the impaired frequencies, treatment course, severity of hearing loss, and different audiometric configurations of SSNHL between the two groups (all $P>0.05$ ). The result showed that diabetic SSNHL patients had a higher level of HbAlc and much worse performance in post-treatment PTA of the impaired frequencies and the number of effective cases, which represented a poor prognosis of SSNHL. The differences were statistically significant (both $P<0.05$ ) (as shown in Table 3). 
TABLE 3 | Prognosis conditions of SSNHL in the matched data set after propensity score matching using prognosis-related factors of SSNHL as the predictors.

\begin{tabular}{|c|c|c|c|}
\hline Indicators & DM group $(n=19)$ & Non-DM group $(n=19)$ & $P$-value \\
\hline \multicolumn{4}{|l|}{ Demographic, clinical, and metabolic variables } \\
\hline Age (years) & $56.00(11.00)$ & $65.00(15.00)$ & 0.389 \\
\hline $\begin{array}{l}\text { Time to treatment initiation } \\
\text { (days) }\end{array}$ & $5.00(5.00)$ & $3.00(6.00)$ & 0.906 \\
\hline $\mathrm{HbA1c}(\%)$ & $7.40(2.65)$ & $5.80(0.40)$ & $<0.001^{*}$ \\
\hline $\begin{array}{l}\text { Pre-treatment PTA of the impaired frequencies } \\
(\mathrm{dBHL})\end{array}$ & $86.43(24.17)$ & $81.43(37.35)$ & 0.139 \\
\hline $\begin{array}{l}\text { Treatment course } \\
\text { (days) }\end{array}$ & $6.08(18.77)$ & $5.00(1.50)$ & 0.472 \\
\hline $\begin{array}{l}\text { Post-treatment PTA of the impaired frequencies } \\
(\mathrm{dBHL})\end{array}$ & $75.83(36.43)$ & $65.00(40.10)$ & $0.032^{*}$ \\
\hline Effective case ( $n, \%)$ & $5(26.3 \%)$ & $11(57.9 \%)$ & $0.049 *$ \\
\hline \multicolumn{4}{|l|}{ Accompanying symptoms $(n, \%)$} \\
\hline Vertigo & $10(52.6 \%)$ & $8(42.1 \%)$ & 0.516 \\
\hline \multicolumn{4}{|l|}{ Severity of hearing loss $(n, \%)$} \\
\hline Mild to moderate & $3(15.8 \%)$ & $6(31.6 \%)$ & 0.447 \\
\hline Severe to profound & $16(84.2 \%)$ & $13(68.4 \%)$ & \\
\hline \multicolumn{4}{|l|}{ Audiometric configurations ( $n, \%)$} \\
\hline LF-SSNHL & $1(5.3 \%)$ & $1(5.3 \%)$ & 0.703 \\
\hline HF-SSNHL & $2(10.5 \%)$ & $2(10.5 \%)$ & \\
\hline FT-SSNHL & $3(15.8 \%)$ & 6 (31.6\%) & \\
\hline TD-SSNHL & 13 (68.4\%) & 10 (52.6\%) & \\
\hline
\end{tabular}

DM, diabetes mellitus; HbA1c, glycosylated hemoglobin A1C; PTA, pure-tone average; SSNHL, sudden sensorineural hearing loss; LF-SSNHL, low-frequency descending type of SSNHL; HF-SSNHL, high-frequency descending type of SSNHL; FT-SSNHL, flat type of SSNHL; TD-SSNHL, total deafnesS of SSNHL.

${ }^{*} p<0.05$ (statistically significant).

The bold values in "Effective cases ( $n, \%)$ " were the number or percentage of SSNHL patients who were classified as effective according to the outcome evaluation criteria.

The bold values in " $P$ values" mean the statistical indicators that were taken as significant when smaller than 0.05.

\section{Correlation Between HbA1c Level and SSNHL}

The level of HbAlc was closely correlated with impaired hearing thresholds, the severity of hearing loss, and different audiometric configurations of SSNHL after PSM (all $P<0.05$ ) (as shown in Table 4). Since the Spearman correlation coefficients of these three parameters were all positive, the result revealed that the higher level of HbAlc in the patients with SSNHL translates to more severe hearing loss as reflected by "TD-SSNHL" dominating the different audiometric configurations of SSNHL, including "LF-SSNHL", "HF-SSNHL", and "FT-SSNHL" (as shown in Table 4). Nevertheless, the prognosis of SSNHL had

TABLE 4 | Correlation between the HbA1c level and SSNHL.

\begin{tabular}{lcc}
\hline Parameters & Spearman $\boldsymbol{r}$ & $\boldsymbol{P}$-value \\
\hline Pre-treatment PTA of the impaired frequencies $^{\mathrm{a}}$ & 0.423 & $\mathbf{0 . 0 0 2}^{\boldsymbol{*}}$ \\
Severity of hearing loss $^{\mathrm{a}}$ & 0.421 & $\mathbf{0 . 0 0 2}^{\boldsymbol{}}$ \\
Audiometric configurations $^{\mathrm{a}}$ & 0.336 & $\mathbf{0 . 0 1 5}^{\star}$ \\
Post-treatment PTA of the impaired frequencies $^{\mathrm{b}}$ & 0.318 & 0.052 \\
Effective case $^{\mathrm{b}}$ & -0.075 & 0.652 \\
\hline
\end{tabular}

PTA, pure-tone average.

${ }^{a}$ We performed a 1:1 match for each patient by using propensity score matching (PSM), choosing "age" as the predictor here.

${ }^{b}$ We performed a 1:1 match for each patient by using PSM, choosing prognosis-related factors of SSNHL as the predictors here.

${ }^{*} p<0.05$ (statistically significant).

The bold values in "Effective cases $(n, \%)$ " were the number or percentage of SSNHL patients who were classified as effective according to the outcome evaluation criteria.

The bold values in "P values" mean the statistical indicators that were taken as significant when smaller than 0.05 . no significant correlation with HbAlc, neither with their posttreatment PTA of the impaired frequencies nor with the number of effective cases (both $P>0.05$ ) (as shown in Table 4).

\section{DISCUSSION}

SSNHL is a sudden illness causing a decrease in hearing of greater than $30 \mathrm{~dB}$ that affects at least three consecutive frequencies within a 72 -h window. Although it effects a wide population, the main pathogenic causes remain unidentified. Among various possible etiologies and pathogenesis, microvascular disorders are the current main suspect linked to the pathophysiological mechanisms proposed for SSNHL. Microangiopathy, which is common in DM patients, happens in multiple organ systems and could also affect the inner ear vessels like the labyrinthine artery. As an artery without collateral compensation, the labyrinthine artery supplies the cochlea, a highly metabolic organ that is extremely sensitive to ischemia and hypoxia $(16,17)$. When chronic or transient microvascular disorders happen in the inner ear, a series of pathophysiological changes would lead to cochlear microcirculation dysfunction and eventually cause hearing loss.

Several histological changes have been observed in preceding related studies that support such hypothesis. In the inner ears of diabetic experimental models or patients, thickening of the basement membrane of the capillaries within the stria vascularis on the lateral walls of the cochlea and narrowing of the internal auditory artery had been found (18-20). Other aspects of the cochlea, such as the loss of Corti organs and 
spiral ganglion neurons and demyelination of the auditory nerve, had also been confirmed in other studies $(21,22)$.

From clinical aspects, studies have clearly demonstrated DM to be one of the risk factors for hearing loss, including SSNHL. A large-sample cohort study in South Korea, after adjusting for multiple potential confounding factors, including demographic characteristics, occupational noise exposure, lifestyle risk factors, and other metabolic abnormalities, found a strong correlation in risk of hearing loss and DM (23). In terms of SSNHL, a recent study that recruited 26,556 patients with DM showed a significant association between DM and an increased risk of developing SSNHL. Meanwhile, such risk was proportional to the severity of DM (24). Another present study demonstrated that the severity of hearing loss in patients with SSNHL was influenced by the presence of DM. A subgroup of SSNHL patients with T2DM was associated with more severe hearing loss (25). In addition, DM was also found to be one of the adverse factors for SSNHL prognosis (26).

Given the abovementioned data, diabetic patients are more likely to suffer sudden deafness and higher severity in hearing loss level and tend to show a poorer prognosis. Furthermore, some research also found that the risk of hearing loss increased progressively with increasing $\mathrm{HbAlc}$ levels above 5\%, suggesting that glucose control may play a role in the development of hearing loss in diabetic patients (23). HbA1c, due to the stability of its Amadori form, is clinically recognized as a better reflection of the plasma glycemic status over the past 2 to 3 months (27). For every 1\% rise in HbA1c, there is a $37 \%$ increase in microvascular diseases (9). Much work has been done to explore the relationship between DM and SSNHL, especially focusing on its microvascular disorders. Until now, few studies to date have analyzed the association between HbA1c and SSNHL.

In this study, we elucidated the association of the HbAlc level with the severity, types, and prognosis of SSNHL in a group of well-defined patients. The clinical and audiometric differences between diabetic and non-diabetic patients with SSNHL were also demonstrated. Among the patients with SSNHL in the present study after PSM, those who met the diagnostic criteria for DM had a higher level of HbAlc and were more severe in their pre-treatment PTA of the impaired frequencies. The proportion of degrees of the hearing loss of the impaired frequencies and audiometric configurations of SSNHL was statistically significant between the two groups. "TD-SSNHL" was dominantly present in SSNHL patients with DM. All these findings indicated that SSNHL patients with DM performed worse in hearing loss at the beginning of SSNHL. The SSNHL prognosis was also worse for diabetic patients. After PSM, more patients in the DM group had a higher post-treatment PTA of the impaired frequencies, and the number of effective cases was less than that in the non-DM group. These results were quite consistent with previous researches.

Based on these findings, we further explored the correlation between the HbA1c level with pre-treatment PTA of the impaired frequencies, severity of hearing loss, different audiometric configurations, and prognosis of SSNHL. We found that the HbAlc level correlated significantly with the first three parameters, which meant that the higher level of HbAlc in the patients with SSNHL, the more serious is the hearing loss in the impaired frequencies pre-treatment, and among all audiometric configurations of SSNHL, "TD-SSNHL" dominated. This revealed a potential connection between SSNHL and glycemic status, suggesting that long-term unsatisfactory blood sugar control may be related to the worse performance in the severity of SSNHL. Considering that a rise in HbA1c meant an increase in microvascular diseases (9), our findings might indirectly support the microvascular etiology hypothesis of SSNHL. It also reminded us that we need to pay close attention to the glucose levels in SSNHL patients and take active measures to control the blood sugar. Given that we found no strong association between the HbAlc level and a prognosis of SSNHL, long-term uncontrolled blood sugar may play a more dominant role in the onset of SSNHL than its progression. The exact mechanism behind it has still not yet been reported. Further work is needed.

There were a few limitations which could be potential sources of bias in this research, including a small sample size limited to a single medical center and measurements of limited parameters. Larger studies containing real-world evidence or multicenter database are required. In addition, the $\mathrm{HbA} 1 \mathrm{c}$ level only represents the long-term blood sugar control (about 2 to 3 months) of the subjects. Whether the immediate and/or short-term level of blood glucose is also related even more sensitively is still unknown. To remedy this, for subsequent studies, we plan to take additional parameters like taking the capillary blood glucose concentrations of patients from fingerstick testing before any treatments and the fasting plasma glucose and glycated albumin level to fully reflect the blood glucose levels of the patients. We will also consider enroling diabetic patients who have not yet developed SSNHL to explore whether blood sugar control is also related to the onset of sudden deafness. A lack of follow-up auditory evaluations was also one of the deficiencies of this study. In the future, we hope to conduct more comprehensive assessments of long-term auditory evaluations to reflect the final hearing condition of patients more accurately.

\section{CONCLUSIONS}

Among patients with SSNHL, diabetic patients had a higher HbAlc level, more severe hearing loss, and poorer prognosis than patients who were without any history of DM. Furthermore, the HbAlc level significantly correlated with the severity of SSNHL, which revealed that the higher the level of HbA1c in the patients with SSNHL, the more serious the hearing loss in the impaired frequencies and among all audiometric configurations of SSNHL, dominated by "TDSSNHL". No strong correlation between the HbA1c level and the prognosis of SSNHL was found in our research. Further studies are needed to determine the molecular mechanisms of 
hyperglycemia and its related microvascular disorders acting on SSNHL.

\section{DATA AVAILABILITY STATEMENT}

The original contributions presented in the study are included in the article/supplementary material. Further inquiries can be directed to the corresponding authors.

\section{ETHICS STATEMENT}

The study protocol was approved and implemented according to the ethical standards of the Shanghai Jiao Tong University Affiliated Sixth People's Hospital ethics committee [2018-KY$036(\mathrm{~K})]$. The trial registration number is ChiCTR18000170072. Written informed consent was obtained before participation. The information of the patients was anonymized and deidentified prior to analysis. The progress was conducted in accordance with the spirit of the Helsinki Declaration.

\section{REFERENCES}

1. Sujana SC, Betty STD, Seth RS, Laura JB, Erynne AF, Sandra AF, et al. Clinical Practice Guideline: Sudden Hearing Loss (Update). Otolaryngol Head Neck Surg (2019) 161(Suppl 1):1-45. doi: 10.1177/0194599819859885

2. Alexander TH, Harris JP. Incidence of Sudden Sensorineural Hearing Loss. Otol Neurotol (2013) 34:1586-9. doi: 10.1097/MAO.0000000000000222

3. Chung JH, Cho SH, Jeong JH, Park CW, Lee SH. Multivariate Analysis of Prognostic Factors for Idiopathic Sudden Sensorineural Hearing Loss in Children. Laryngoscope (2015) 125:2209-15. doi: 10.1002/lary.25196

4. Rust KR, Prazma J, Triana RJ, Michaelis OE, Pillsbury HC. Pillsbury, Inner Ear Damage Secondary to Diabetes Mellitus. II. Changes in Aging SHR/N-Cp Rats. Arch Otolaryngol Head Neck Surg (1992) 118:397-400. doi: 10.1001/ archotol.1992.01880040059010

5. American Diabetes Association. 2. Classification and Diagnosis of Diabetes: Standards of Medical Care in Diabetes-2020. In: Diabetes Care, vol. 43. San Francisco: American Diabetes Association (2020). p. 14-31. doi: 10.2337/dc20-S002

6. Lenters-Westra E, Schindhelm RK, Bilo HJ, Slingerland RJ. Haemoglobin Alc: Historical Overview and Current Concepts. Diabetes Res Clin Pract (2013) 99:75-84. doi: 10.1016/j.diabres.2012.10.007

7. Chen B, Bestetti G, Day RM, Turner APF. The Synthesis and Screening of a Combinatorial Peptide Library for Affinity Ligands for Glycosylated Haemoglobin. Biosensors Bioelectronics (1998) 13:779-85. doi: 10.1016/ S0956-5663(98)00042-6

8. Goldstein DE, Little RR, Lorenz RA, Malone JI, Nathan D, Peterson CM, et al. Tests of Glycemia in Diabetes. Diabetes Care (2004) 27:1761-73. doi: 10.2337/ diacare.27.7.1761

9. Stratton IM, Adler AI, Neil HA, Matthews DR, Manley SE, Cull CA, et al. Association of Glycaemia With Macrovascular and Microvascular Complications of Type 2 Diabetes (UKPDS 35): Prospective Observational Study. BMJ (2000) 321:405-12. doi: 10.1136/bmj.321.7258.405

10. Expert Committee on the Diagnosis and Classification of Diabetes Mellitus. Report of the Expert Committee on the Diagnosis and Classification of Diabetes Mellitus. Diabetes Care (2003) 26(Suppl 1):5-20. doi: 10.2337/diacare.26.2007.s5

11. World Health Organization. Future Programme Developments for Prevention of Deafness and Hearing Impairments: Report of the First Informal Consultation. Geneva (1997).

12. Editorial Board of Chinese Journal of Otolaryngology Head and Neck Surgery, The Otolaryngology Head and Neck Surgery Branch of Chinese Medical Association. Guideline of Diagnosis and Treatment of Sudden Deafness

\section{AUTHOR CONTRIBUTIONS}

YF and ZheZ have given substantial contributions to the conception and the design of there search. YS and ZhoZ contributed to the acquisition, analysis, and interpretation of the data. All authors have participated in drafting the manuscript. YF, ZheZ and DQ revised it critically. All authors contributed to the article and approved the submitted version.

\section{FUNDING}

This research was funded by a grant from International Cooperation and Exchange of the National Natural Science Foundation of China (NSFC; 81720108010) to SY and the National Natural Science Foundation of China (no. 81771015) to YF.

\section{ACKNOWLEDGMENTS}

The authors thank all the participants of the study.

(2015). Chin J Otorhinolaryngol Head Neck Surg (2015) 50:443-7. doi: 10.3760/cma.j.issn.1673-0860.2015.06.002

13. Stachler RJ, Chandrasekhar SS, Archer SM, Rosenfeld RM, Schwartz SR, Barrs DM, et al. Clinical Practice Guideline: Sudden Hearing Loss. Otolaryngol Head Neck Surg (2012) 146(Suppl 3):1-35. doi: 10.1177/ 0194599812436449

14. Fetterman BL, Saunders JE, Luxford WM. Prognosis and Treatment of Sudden Sensorineural Hearing Loss. Am J Otol (1996) 17:529-36.

15. Haynes DS, O'Malley M, Cohen S, Watford K, Labadie RF. Intratympanic Dexamethasone for Sudden Sensorineural Hearing Loss After Failure of Systemic Therapy. Laryngoscope (2007) 117:3-15. doi: 10.1097/ 01.mlg.0000245058.11866.15

16. Lin CF, Lee KJ, Yu SS, Lin YS. Effect of Comorbid Diabetes and Hypercholesterolemia on the Prognosis of Idiopathic Sudden Sensorineural Hearing Loss. Laryngoscope (2016) 126:142-9. doi: 10.1002/lary.25333

17. Orita S, Fukushima K, Orita Y, Nishizaki K. Sudden Hearing Impairment Combined With Diabetes Mellitus or Hyperlipidemia. Eur Arch Otorhinolaryngol (2007) 264:359-62. doi: 10.1007/s00405-006-0196-6

18. Costa OA. Inner Ear Pathology in Experimental Diabetes. Laryngoscope (1967) 77:68-75. doi: 10.1288/00005537-196701000-00005

19. Fukushima H, Cureoglu S, Schachern PA, Paparella MM, Harada T, Oktay MF. Effects of Type 2 Diabetes Mellitus on Cochlear Structure in Humans. Arch Otolaryngol Head Neck Surg (2006) 132:934-8. doi: 10.1001/archotol.132.9.934

20. Kakarlapudi V, Sawyer R, Staecker H. The Effect of Diabetes on Sensorineural Hearing Loss. Otol Neurotol (2003) 24:382-6. doi: 10.1097/00129492200305000-00006

21. Raynor E, Robison WG, Garrett CG, McGuirt WT, Pillsbury HC, Prazma J. Consumption of a High-Galactose Diet Induces Diabetic-Like Changes in the Inner Ear. Otolaryngol Head Neck Surg (1995) 113:748-54. doi: 10.1016/ S0194-5998(95)70015-3

22. Nakae S, Tachibana M. The Cochlea of the Spontaneously Diabetic Mouse. II. Electron Microscopic Observations of Non-Obese Diabetic Mice. Arch Otorhinolaryngol (1986) 243:313-6. doi: 10.1007/BF00460208

23. Kim MB, Zhang Y, Chang Y, Ryu S, Choi Y, Kwon MJ, et al. Diabetes Mellitus and the Incidence of Hearing Loss: A Cohort Study. Int J Epidemiol (2017) 46:717-26. doi: 10.1093/ije/dyw243

24. Lin SW, Lin YS, Weng SF, Chou CW. Risk of Developing Sudden Sensorineural Hearing Loss in Diabetic Patients: A Population-Based Cohort Study. Otol Neurotol (2012) 33:1482-8. doi: 10.1097/MAO. ob013e318271397a 
25. Fukui M, Kitagawa Y, Nakamura N, Kadono M, Mogami SI, Ohnishi M, et al. Idiopathic Sudden Hearing Loss in Patients With Type 2 Diabetes. Diabetes Res Clin Pract (2004) 63:205-11. doi: 10.1016/j.diabres.2003.09.013

26. Zhou YL, Qiu SY, Liu DB. Impact of Metabolic Syndrome on Recovery of Idiopathic Sudden Sensorineural Hearing Loss. Am J Otolaryngol (2019) 40:573-6. doi: 10.1016/j.amjoto.2019.05.011

27. Ang SH, Thevarajah M, Alias Y, Khor SM. Current Aspects in Hemoglobin Alc Detection: A Review. Clin Chim Acta (2015) 439:202-11. doi: 10.1016/ j.cca.2014.10.019

Conflict of Interest: The authors declare that the research was conducted in the absence of any commercial or financial relationships that could be construed as a potential conflict of interest.
Publisher's Note: All claims expressed in this article are solely those of the authors and do not necessarily represent those of their affiliated organizations, or those of the publisher, the editors and the reviewers. Any product that may be evaluated in this article, or claim that may be made by its manufacturer, is not guaranteed or endorsed by the publisher.

Copyright (C) 2021 Shen, Zheng, Xiao, Liu, Guo, Chen, Wu, Shi, Zhang, Qian, Feng and Yin. This is an open-access article distributed under the terms of the Creative Commons Attribution License (CC BY). The use, distribution or reproduction in other forums is permitted, provided the original author(s) and the copyright owner(s) are credited and that the original publication in this journal is cited, in accordance with accepted academic practice. No use, distribution or reproduction is permitted which does not comply with these terms. 